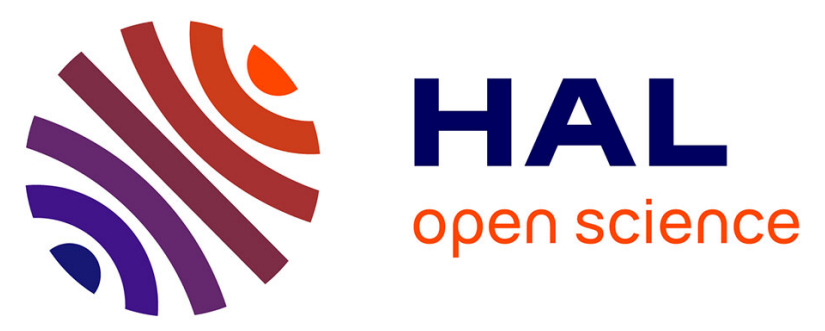

\title{
Hierarchical Intelligent Component-Based Development for the Design of Cyber-Physical Control Architecture
}

Alexandre Parant, François Gellot, Philippot Alexandre, Véronique Carré-Ménétrier

\section{- To cite this version:}

Alexandre Parant, François Gellot, Philippot Alexandre, Véronique Carré-Ménétrier. Hierarchical Intelligent Component-Based Development for the Design of Cyber-Physical Control Architecture. International Conference on Control and Fault-Tolerant Systems (SysTol), 2021, Saint-Raphaël, France. 10.1109/SysTol52990.2021.9596041 . hal-03427345

\section{HAL Id: hal-03427345 \\ https://hal.science/hal-03427345}

Submitted on 13 Nov 2021

HAL is a multi-disciplinary open access archive for the deposit and dissemination of scientific research documents, whether they are published or not. The documents may come from teaching and research institutions in France or abroad, or from public or private research centers.
L'archive ouverte pluridisciplinaire HAL, est destinée au dépôt et à la diffusion de documents scientifiques de niveau recherche, publiés ou non, émanant des établissements d'enseignement et de recherche français ou étrangers, des laboratoires publics ou privés. 


\title{
Hierarchical Intelligent Component-Based Development for the Design of Cyber-Physical Control Architecture
}

\author{
Alexandre Parant, François Gellot, Alexandre Philippot and Véronique Carré-Ménétrier
}

\begin{abstract}
Modern manufacturing systems are subject to many disruptions due to increased competition. They are subject to many configuration changes during their life cycle and must cost-effectively make these changes. Intelligent componentbased development enables the rapid design of control systems. This paper presents a new methodology for structuring the control architecture of cyber-physical systems with intelligent components: The Hierarchical Intelligent Component Architecture (HICA). Compared to traditional design methods, this methodology integrates the design of the physical and software architecture simultaneously. This paper also describes the model-driven engineering to realize this architecture using SysML diagrams to implement the code in IEC 61499 standard. Diagram Linkage Table (DTL) is the central element that allows the transition from SysML diagrams to IEC 61499.
\end{abstract}

\section{INTRODUCTION}

Cyber-Physical Systems (CPS) operate in an unpredictable environment and are subject to many voluntary and involuntary changes. The adaptability, connectivity, and distribution of the control system are characteristics that allow the system to adapt to the constant evolution of products and consumer needs [1]. In CPSs, embedded systems control and supervise physical processes. In this context, the design of the control system becomes more and more complex. New requirements have to be satisfied [2] :

- Portability: Software tools can interpret components produced by other software.

- Configurability: Software tools can configure a device from different vendors.

- Interoperability: Embedded systems can cooperate to perform operations through distributed applications.

- Reconfigurability: The hardware and its control software are adaptable during operation.

- Distributivity: Software components can be distributed on different devices.

Traditional design methods do not consider these specificities and the links between the physical and virtual worlds. The design of the control system does not start until the design of the physical system is finished, which leads to sub-optimal solutions [3]. Various works have been carried out on Intelligent Mechatronic Components (IMC), combining the software part and the physical part of a component [4]. IMCs allow several disciplines to be brought together in a single component, thus improving engineering efficiency [5]. Indeed, the common structure allows to link the different

Authors work at the Centre de Recherche en Sciences et Technologies de l'Information et de la Communication (CReSTIC), University of Reims disciplines; this avoids redundancy, loss, and inconsistency of information.

This overview has consequences for the control system. The control point of view should not be limited to the automation point of view; the software and physical parts of an installation are closely linked. Therefore, it is necessary to have a higher-level vision and use different views to understand the growing complexity of these systems.

Thramboulidis [6] demonstrates this necessity by developing simultaneous engineering of the different components making up the system. A mechanical part, an electrical part, and a software part constitute the component. A multi-layer architecture is developed, the components are assembled in one layer, and then they are projected vertically in the other layers. Each layer models a specific functionality of the system (mechanical composition, software architecture, and control software).

One of the problems is to link different domains. Each domain has its software and its tools. Transformation models are defined to convert Domain-Specific Models (DSM) into the SysML (System Modelling Language) profile model in [7]. The common model allows to highlight dependencies and to maintain consistency between domains. Our proposal is to model the knowledge of the system through SysML diagrams and to link the whole in a table. The coherence of the information is ensured by a central element and not by a multitude of transformation models, which limits errors.

The main objective of this paper is to define a hierarchical structure of the control through intelligent elements to obtain a structure like the plant's physical architecture. The architecture defines a specific structure for the intelligent elements according to their hierarchical level. In addition, a model-based design is proposed to develop the control system. The models are used to define the customer's requirements and the structural and behavioral aspects of the system. One of the contributions is the Diagram Linkage Table (DLT) that allows linking the high-level diagrams that describe the knowledge of the system with the low-level control system. IEC 61499 standard implements the low-level control. Another contribution is the use of a knowledge base containing interconnected diagrams to reconfigure the system efficiently and quickly during a disturbance.

Section 1 showed the need for a system overview to facilitate the engineering of the control system in CPS. Section 2 focuses on intelligent components and presents the IEC 61499 standard and SysML for system modeling. Section 3

Champagne-Ardenne, Reims, France. (e-mail: \{alexandre.parant, francois.gellot, alexandre.philippot, veronique.carre $\} @$ univ-reims.fr). 
gives the proposed architecture for structuring the CPS control architecture using intelligent components. It presents the design steps linked with the diagrams to obtain this structure and it presents the advantages when the system needs to be reconfigured. Section 4 presents an implementation on a simulated system.

\section{DESIGN BASED ON INTELLIGENT COMPONENTS}

\section{A. Intelligent Mechatronic Component}

IMCs allow the use of the component-based design (CBD) paradigm to design the control system. The functionality defines the component; they have predefined communication interfaces to assemble them. They have a certain autonomy and cooperate with other components of the system to reach a common goal.

Vyatkin introduces in [4] the term Intelligent Mechatronics Component (IMC). The components of a system are no longer only mechanical devices; they also embed their control system and additional data.

In [8], the authors present a control block design method for a reusable IMC based on functionality and its perception of its environment. They integrate these components into different control architectures to compare the overall performance.

The authors in [5] use the term automation components, including the basic control of the component, a diagnostic module, an Human Machine Interface (HMI) module, and a universal interface module for communication between the components. They also define the assembly and interactions between the components to form a hierarchical structure to base the control structure on the system's physical structure. The authors add a test subcomponent and a condition monitoring subcomponent in the automation component in [9]. These subcomponents allow components to know the state of other components and test them after changes.

In [10], the authors propose a hierarchical control architecture based on automation components. The components integrate control and interface subcomponents. The ends of the components are the communication interface. The front-end of a component interacts with the back end of another component to form a hierarchical structure.

Black and Vyatkin extend the Model-View-Controller (MVC) paradigm to predict the future state of the component in [11]. The intelligent component consists of a dynamic model of the system and an interface connecting it to the components' actual sensors and actuators. The prediction module uses actual or simulated values depending on the operating mode.

The disadvantage of the proposed approaches is that the intelligent components all have the same structure. The approaches propose a hierarchical architecture of the control system that is not reflected in the intelligent components. Lowlevel components do not have the same reflection capabilities as high-level components. The components have the same sub-modules, but these will have different structures depending on their intelligence.
Intelligent components allow the development of a component-based controller. The advantage is rapid development without rewriting code, and components can be verified and validated upstream to be put in a library. The redesign phase is not necessary to use components in a multitude of different applications. The organization of a hierarchy in intelligent components makes it possible to create a library at different levels. The reuse of components can go from a single component to an entire cell.

The control structure becomes like the physical and functional structure of the plant. This idea corresponds to the CPSs' philosophy which aims to link the physical part to the logical part. The system becomes more robust than a monolithic control system, a failure on a component does not propagate to the whole control system. A diagnostic module can be attached to the component to solve minor failures. The control system is modular. Integration or conversion of a component is possible if it has the corresponding physical and functional interfaces. The system can be reconfigured online without stopping production.

Traditional control systems in a manufacturing system are on Programmable Logic Controllers (PLCs) and IEC6131-3 languages-centric. The control system is monolithic and centralized. It is not easy to modify, which is not compatible with the use of IMC. The following subsection presents the IEC 61499 standard, which is promising for designing the IMC software part. It has many advantages for the design and validation of model-based controls [12].

\section{B. IEC 61499}

The IEC 61499 standard [13] enables the design and development of distributed control systems [14]. Compared to the traditional IEC 61131-3 standard with a program block that repeats in a loop, IEC 61499 is composed of function blocks incorporating code and is event driven. The arrival of an event at the input of a function block triggers the code contained in the block. One of the advantages of function blocks is the principle of encapsulation which allows the safe reuse of blocks already used in a previous program. The inter-module communication is done only by sending messages, which ensures the independence of the internal variables of each block. Function blocks represent each one a process that facilitates the implementation and the modifications of the components. Function blocks assembly allows designing the control system. The network of function blocks can then be distributed among several controllers interconnected by communication tools while keeping its behavior [15].

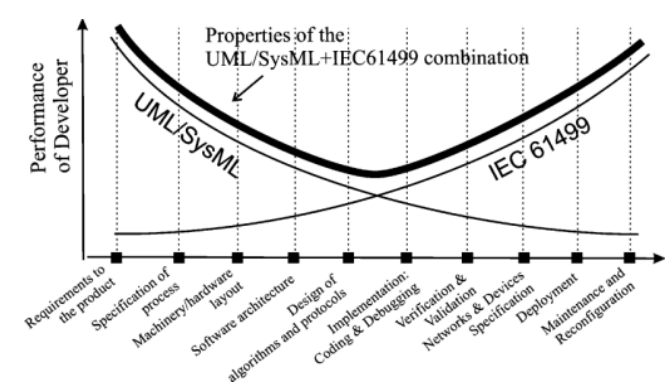

Figure 1. Combinaison of SysML and IEC 61499 [16] 
IEC 61499 is therefore suitable for machine control in CPS. It has interesting features for the reconfigurable part of the control due to its process thinking and structure, allowing modular construction and easier integration or conversion of a component.

Despite the use of function blocks, a control system defined in IEC 61499 remains challenging to read. Multiple threads connect the blocks, and the code quickly becomes unreadable as the system becomes complex and the network of events and data overlap. The methodology described in section 3 proposes using a design methodology based on SysML diagrams and defining the system in a first step according to different views to facilitate writing the code in IEC 61499.

\section{Systems Modeling Language}

IEC 61499 allows a precise definition of the control architecture. The control system is divided between several devices that can be divided into resources. Each function block has its variables allowing a complete distribution of the control. These characteristics are interesting for the deployment of control at the controller level. The difficulty lies in the control design. The association with SysML/UML diagrams makes it possible to fill the gaps in IEC 61499 in the first stages of design (see Fig. 1)

Systems Modeling Language (SysML) allows to model systems from different points of view thanks to a multitude of diagrams. It is an extension of UML 2.0 [16]. It supports the design, verification, and validation of complex systems. SysML has a broader view of the system, and it has a higherlevel view. The diagrams can include hardware, software, data, and personnel. These features are used in [17] for the implementation of the control. The software part described in UML is extracted from the SysML system model and then refined to obtain the code. The methodology described in the next section applies the same principles. A hierarchical architecture based on intelligent components structures the control system. A set of SysML diagrams models this architecture thanks to different high-level viewpoints. A table links all the domains, linking all the information to the system at the control.

\section{MODEL-BASED ENGINEERING FOR CONTROL ARCHITECTURE DESIGN}

This section presents a new control architecture for CPS. This approach hierarchically structures IMCs to link the control architecture to the physical architecture. A top-down design methodology is presented to start from the high-level knowledge of the system to low-level control code. The central element is the Diagram Linkage Table (DLT) that links the SysML diagrams to the code implemented in IEC 61499.

\section{A. Hierarchical Intelligent Component Architecture (HICA)}

Fig. 2 shows the elements that make up each of the system components. Indeed, compared to other approaches based on IMC, the internal structure of the elements is different depending on the hierarchical level of the system. The internal structure adapts to the intelligence available thanks to the computing power of the controller at each level. Four

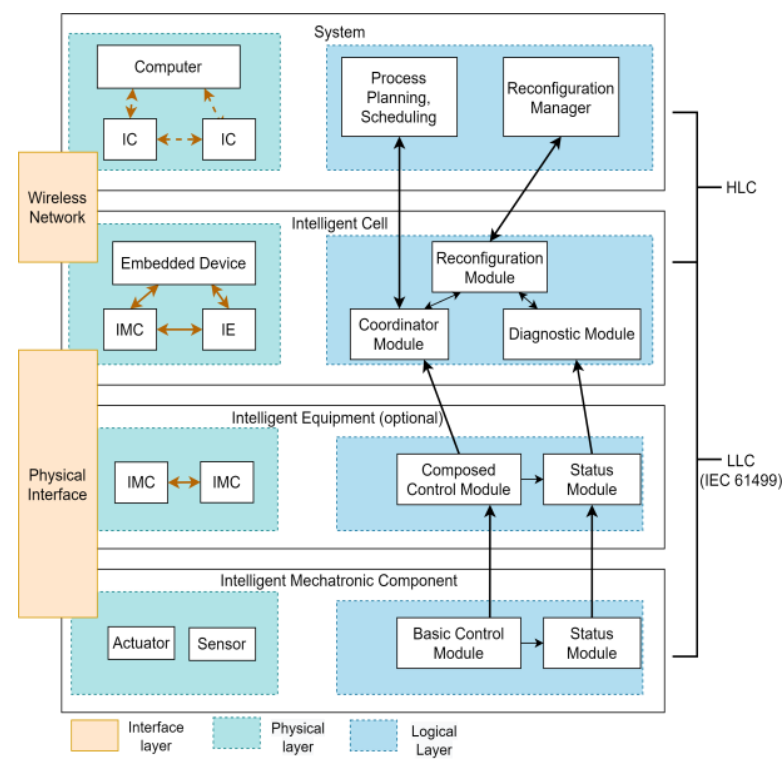

Figure 2. Hierarchical Intelligent Component Architecture.

hierarchical intelligent levels compose HICA: (1) System, (2) Intelligent Cell, (3) Intelligent Equipment, and (4) Intelligent Mechatronic Component.

(1) The system is at the highest level, and this paper does not discuss it in detail. Its role is to optimize the production in a normal state and send the necessary orders to the cells during a configuration change. Wireless networks do the communication between the system and the cells. The communication protocol is still to be determined.

(2) The Intelligent Cell (IC) is composed of several Intelligent Equipment (IE) and Intelligent Mechatronic Components (IMC). IC provides the ability to perform more complex operations offered as services to the system layer. The assembly of the IE and IMC allows designing the code semi-automatically. However, the designer will need to add coordination elements between the basic and compound control modules according to production specifications to achieve the desired functionality. Components provide basic operations, and it is necessary to coordinate these operations to perform complex tasks. The coordination elements come in to resolve coordination problems. For example, a component may be at the intersection of two conveyors, and a coordination module is needed to manage the priority in the use of this component. Performance or energy management specifications can determine the priority, for example.

The IC logic part is composed of three components:

- The coordinator module ensures the coordination between the different equipment according to the planning sent by the system layer. It ensures the success of the process.

- The diagnostic module retrieves the states of the sublevel elements. It ensures that there is no failure in the cell. In case of failure, it can notify the reconfiguration module, which will notify the system layer if it cannot replace the fault. 
- The reconfiguration module links to the system layer when a reconfiguration appears. It can handle a minor reconfiguration locally in case of failure, especially a software failure of the equipment. However, its primary role is to translate the reconfiguration orders sent by the system layer and apply them to the coordinator while keeping the system safe to operate.

(3) Intelligent Equipment (IE) and (4) Intelligent Mechatronic Components (IMC) have a similar internal structure. The hierarchical decomposition of the system described in the following subsection differentiates these two elements. IE is an aggregation of IMCs, the control module allows more complex operations than a component, but the state module has the same functionality. Intelligent components must have a perception of their internal state. A status module monitors its status and warns the upper layer in case of failure. Embedded systems control IE and IMC. The structure between the different devices is identical, allowing different devices to be stored in a library to design the cell by a drag and drop system.

\section{B. Hierarchical decomposition of the plant}

The determination of the system elements is done hierarchically (see Fig.2). The objective is to define the objectoriented structure of a system as well as possible. The basic elements defined are the components: the component has at least one sensor or actuator. It can have several sensors or actuators. The component performs only simple operations. For example, a conveyor is used to transport an object with the system, so it will be considered a component.

An Equipment can perform more complex operations than a single piece of component. Physical and functional interactions determine the assembly of components into equipment. For example, an actuator located on a conveyor is physically linked to the conveyor. It is dependent on the conveyor to existing in the system. The cylinder extends the

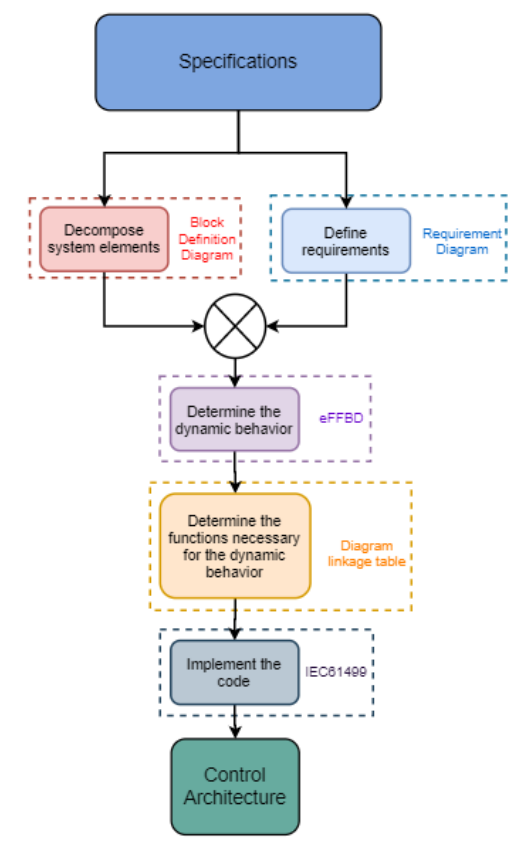

Figure 3. Model-based engineering for HICA. capacities of the conveyor. The two work together, so their combination creates equipment.

The cell is a grouping of equipment; it allows to carry out assemblies or produce a part. The cell can work autonomously. It can be integrated into a system to realize a range of products.

This control architecture allows having components with clearly established limits to define the global system best. The control architecture takes the object-oriented structure of the physical structure of the system. The design or redesign of the control system following a configuration change is facilitated. It will be sufficient to identify the element(s) impacted by the change and to replace them with components having the same physical and functional characteristics. The SysML diagrams presented in the following subsection allow components to be linked to a specific requirement to identify the distribution of tasks in the control system.

\section{Model-based engineering for HICA}

In addition to proposing different internal architectures for the intelligent components, a set of diagrams describes the system in the proposed methodology (see Fig. 3). The diagrams represent the system as a whole and facilitate the control design. They model the behavior and knowledge of the system and will be used in future work to facilitate the reconfiguration of the system at different levels.

Each diagram offers a different point of view to model the knowledge and behavior of the system. The Block Definition Diagram (BDD) and the Requirement Diagram (Req) are among SysML diagrams. BDD allows to specify the system's physical hierarchical decomposition and have a global view of the operations proposed by each component of the system. Req allows decomposing the specifications of the specifications. It can include functional, performance, and security specifications. These two diagrams are designed simultaneously because each one influences the other: Req specifies which requirement is satisfied by which element of the system. This link allows the designer to have a broad knowledge of the system. He knows what purpose each component serves. The following diagrams will define what operation the element must do to satisfy the requirement.

The enhanced Function Flow Block Diagram (eFFBD) models the dynamic behavior of the system. eFFBD models control flows between functions, as well as data exchanges. This diagram is ideal for modeling the dynamic behavior of an IEC 61499 network. Control flows between functions represent events between function blocks. In addition,

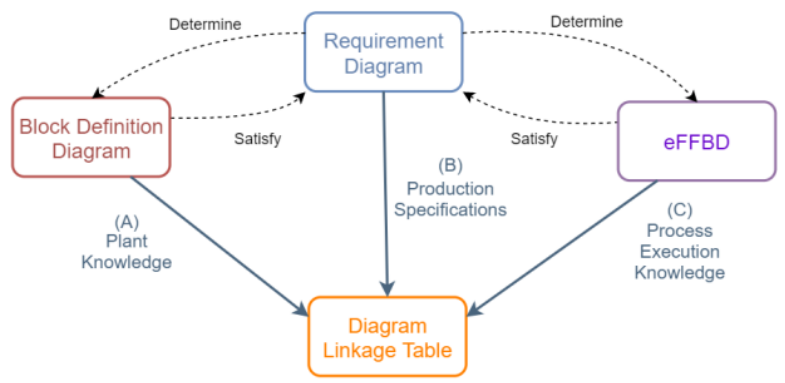

Figure 4. Interdependency in knowledge base. 
modeling the data flows in parallel allows for a structure like the IEC 61499 code. Modeling in eFFBD allows for a higherlevel language with function blocks described by sentences. It is more understandable than an IEC 61499 network. It is possible to use the activity diagram defined in SysML instead of eFFBD because it also allows for model actions, flows, and conditions of actions. This step is done in a recursive way using Req. The designer first tries to satisfy the requirements at the top of the hierarchy before going down to the lowest level requirements until he gets a description as close as possible to the IEC 61499 network.

The specifications define the physical components and the order of execution of the processes. Req, BDD, and eFFBD define the high-level knowledge of the system from three perspectives: (A) plant knowledge, (B) production specifications, and (C) process execution knowledge (see Fig. 4). The Diagram Linkage Table (DLT) is the central model of the approach; it links the specifications, the physical structure, and the system's dynamic behavior. It links the abstract highlevel description of the system to the low-level control in IEC 61499 function blocks. DLT groups the requirements of the Req diagram, the functions of the eFFBD diagram, and the operations proposed by the control module of the IEs and IMCs composing the system. These links allow the generation of a part of the IEC6199 FB network without writing code.

\section{Reconfiguration abilities}

The use of the knowledge base has two interests: the first described above during the design of the control architecture and the second during the life cycle of the system. The interconnection of the parts of the knowledge base improves the reconfiguration capabilities. The designer can then quickly estimate the impact of a disturbance on the whole system and adopt an adequate response.

Our methodology reduces the time required to change the configuration of the control system by (1) identifying the
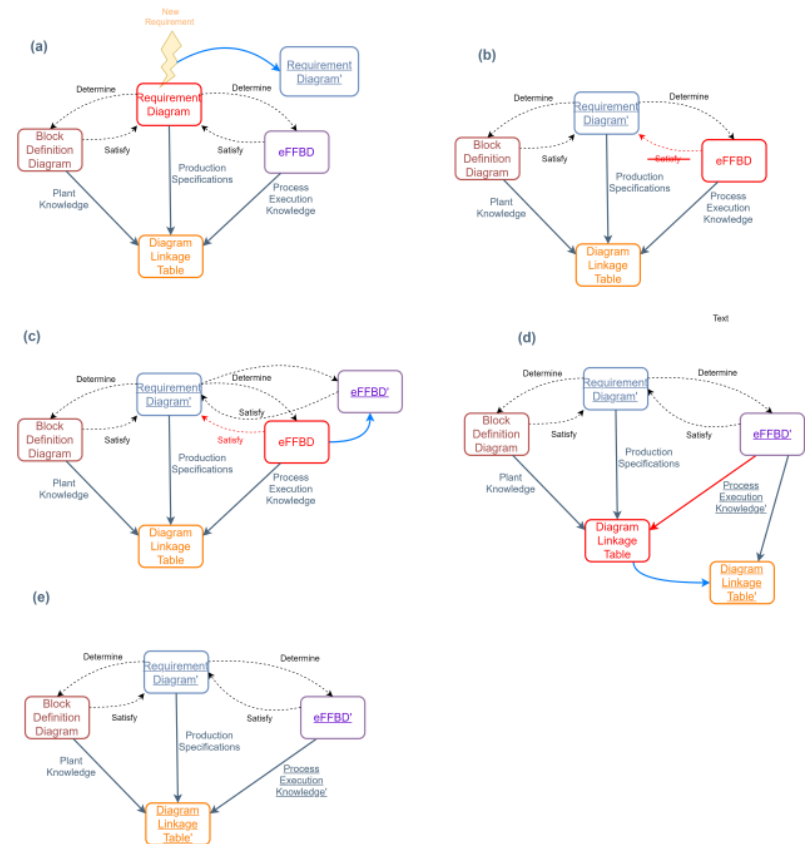

Figure 5. Reconfiguration of knowledge base.

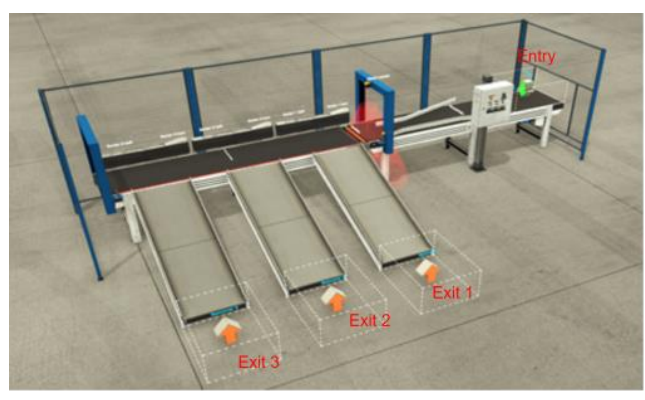

Figure 6. Sorting station in Factory I/O.

corrupted components, (2) impacting the code through DLT, and (3) providing the designer with a set of proposed solutions.

The following example shows a change in the knowledge base due to a change in specifications. The perturbation does not require changing the physical structure of the system. The solution is then to rearrange the program connections to modify the dynamic behavior of the system to meet the new specifications. The designer should perform the following procedure to reconfigure the system in this specific case (see Fig. 5):

(a) A new specification comes to express a new need from the customer: The designer adds the specification to the old requirements diagram to give the Req' diagram.

(b) The following case evokes the simplest solution when there are only one of the two diagrams impacted by the change of specifications, here it is the eFFBD which does not satisfy Req'.

(c) The designer must modify the eFFBD to satisfy the new requirements diagram.

(d) eFFBD' satisfies Req' but the production knowledge changes due to the change in the system behavior, the DLT must be updated.

(e) The designer updates the table to obtain TLD' and will need to reflect the changes in the code.

\section{USE CASE}

The methodology presented was implemented on a simulated manufacturing system using Factory I/O software (see Fig. 6). There are three types of parts that pass through the system. The requirements of the specification are (i) control of the system by an operator, (ii) identification of the part, and (iii) transport of the part to the appropriate exit.

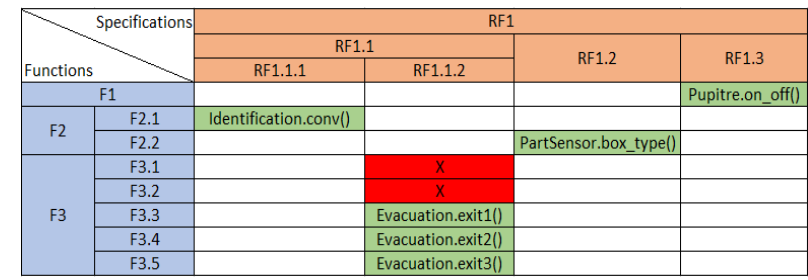

Figure 7. Diagram Linkage Table of sorting station. 
Fig. 7 shows the DLT obtained by following the design steps. The rows of the table are the identifiers of the functions defined in the eFFBD and the columns are the identifiers of the requirements defined in the Req diagram. Each cell of the DLT must be filled by an operation of the system that satisfies the requirement of the column and that is described by the function of the row. The crosses in the table correspond to operations that are not proposed by the system components. They can be coordination between two independent IMCs or IEs of the system. The designer will have to develop IEC61499 FB containing the corresponding logic.

This example shows the feasibility of the methodology for the design of the control architecture. The transition to the IEC61499 standard is achieved by replacing the eFFBD functions with the IEC61499 FB contained in the components.

\section{CONCLUSION}

This paper presents a new methodology for structuring the control architecture of a CPS. We have structured the control architecture as a hierarchy of intelligent components to represent different views of the system in a common model. The elements can be grouped in a library according to several levels to build a system on the principle of plug and produce. Each component has a different level of intelligence depending on the available computing power. This decomposition allows for a more granular structuring of the system intelligence to approach the 5C architecture [18].

We use the SysML diagram to perform the specification and control synthesis phase and the IEC61499 standard to implement the control at the lowest levels. The software part is associated with the production and physical installation specifications. The control is flexible and fault tolerant. Diagram Linkage Table (DLT) reflects the changes in one domain in the others. The influence of this perturbation is then visible, and the designer sees the parts of the code to be modified to satisfy the new conditions. The analysis of the impact of a disturbance allows to determine the impacted elements. The configuration change is then precisely targeted, and time is reduced.

Future work will include developing an ontology for each diagram of the system and the reconfiguration orders. A clear view provides understandable knowledge of the system and its interaction. Creating a common language will greatly facilitate interactions between Low-Level Control (LLC) and HighLevel Control (HLC). The reconfiguration processes could be done without the help of a human operator. On the one hand, LLC could warn HLC of a physical or software failure of a component. On the other hand, HLC could then react thanks to its knowledge of the system and send a reconfiguration order directly interpretable by LLC thanks to a translation in IEC 61499.

\section{ACKNOWLEDGMENT}

This work is integrated into the project FFCA (Factories of Future Champagne-Ardenne). The authors would like to thank the region Grand-Est within the project FFCA (CPER PFEXCEL).

\section{REFERENCES}

[1] L. Monostori et al., "Cyber-physical systems in manufacturing," CIRP Annals, vol. 65, no. 2, pp. 621-641, Jan. 2016, doi: 10.1016/j.cirp.2016.06.005.

[2] T. Strasser, A. Zoitl, J. H. Christensen, and C. Sünder, "Design and Execution Issues in IEC 61499 Distributed Automation and Control Systems," IEEE Transactions on Systems, Man, and Cybernetics, Part C (Applications and Reviews), vol. 41, no. 1, pp. 41-51, Jan. 2011, doi: 10.1109/TSMCC.2010.2067210.

[3] F. Mhenni, J.-Y. Choley, O. Penas, R. Plateaux, and M. Hammadi, “A SysML-based methodology for mechatronic systems architectural design," Advanced Engineering Informatics, vol. 28, no. 3, pp. 218231, Aug. 2014, doi: 10.1016/j.aei.2014.03.006.

[4] V. Vyatkin, "Intelligent mechatronic components: control system engineering using an open distributed architecture," in EFTA 2003. 2003 IEEE Conference on Emerging Technologies and Factory Automation. Proceedings (Cat. No.03TH8696), Sep. 2003, vol. 2, pp. 277-284 vol.2. doi: 10.1109/ETFA.2003.1248711.

[5] C. Sunder, A. Zoitl, and C. Dutzler, "Functional structure-based modelling of automation systems," International Journal of Manufacturing Research, vol. 1, no. 4, pp. 405-420, Jan. 2006, doi: 10.1504/IJMR.2006.012253.

[6] K. Thramboulidis, "Model-integrated mechatronics - toward a new paradigm in the development of manufacturing systems," IEEE Transactions on Industrial Informatics, vol. 1, no. 1, pp. 54-61, Feb. 2005, doi: 10.1109/TII.2005.844427.

[7] A. A. Shah, A. A. Kerzhner, D. Schaefer, and C. J. J. Paredis, "Multiview Modeling to Support Embedded Systems Engineering in SysML," in Graph Transformations and Model-Driven Engineering: Essays Dedicated to Manfred Nagl on the Occasion of his 65th Birthday, G. Engels, C. Lewerentz, W. Schäfer, A. Schürr, and B. Westfechtel, Eds. Berlin, Heidelberg: Springer Berlin Heidelberg, 2010, pp. 580-601. doi: 10.1007/978-3-642-17322-6_25.

[8] M. Sorouri, S. Patil, and V. Vyatkin, "Distributed control patterns for intelligent mechatronic systems," in IEEE 10th International Conference on Industrial Informatics, Jul. 2012, pp. 259-264. doi: 10.1109/INDIN.2012.6301149.

[9] R. Hametner, A. Zoitl, and M. Semo, "Automation component architecture for the efficient development of industrial automation systems," in 2010 IEEE International Conference on Automation Science and Engineering, Aug. 2010, pp. 156-161. doi: 10.1109/COASE.2010.5584013.

[10] G. Cengic, O. Ljungkrantz, and K. Akesson, “A Framework for Component Based Distributed Control Software Development Using IEC 61499," in 2006 IEEE Conference on Emerging Technologies and Factory Automation, Sep. 2006, pp. 782-789. doi: 10.1109/ETFA.2006.355186.

[11] G. Black and V. Vyatkin, "Intelligent Component-Based Automation of Baggage Handling Systems With IEC 61499," IEEE Transactions on Automation Science and Engineering, vol. 7, no. 2, pp. 337-351, Apr. 2010, doi: 10.1109/TASE.2008.2007216.

[12] C. Yang, V. Vyatkin, and C. Pang, "Model-Driven Development of Control Software for Distributed Automation: A Survey and an Approach," IEEE Transactions on Systems, Man, and Cybernetics: Systems, vol. 44, no. 3, pp. 292-305, Mar. 2014, doi: 10.1109/TSMCC.2013.2266914.

[13] IEC 61499 Function Blocks - Part 1: Architecture, Edition. 2.0.

[14] V. Vyatkin, "IEC 61499 as Enabler of Distributed and Intelligent Automation: State-of-the-Art Review," IEEE Transactions on Industrial Informatics, vol. 7, no. 4, pp. 768-781, Nov. 2011, doi: 10.1109/TII.2011.2166785.

[15] C. Pang, S. Patil, C. Yang, V. Vyatkin, and A. Shalyto, "A portability study of IEC 61499: Semantics and tools," in 2014 12th IEEE International Conference on Industrial Informatics (INDIN), Jul. 2014, pp. 440-445. doi: 10.1109/INDIN.2014.6945553.

[16] M. Hause, "The SysML Modelling Language," 2006.

[17] K. Thramboulidis, "A cyber-physical system-based approach for industrial automation systems," Computers in Industry, vol. 72, pp. 92-102, Sep. 2015, doi: 10.1016/j.compind.2015.04.006.

[18] J. Lee, B. Bagheri, and H.-A. Kao, "A Cyber-Physical Systems architecture for Industry 4.0-based manufacturing systems," Manufacturing Letters, vol. 3, pp. 18-23, Jan. 2015, doi: 10.1016/j.mfglet.2014.12.001. 